Editorial

\title{
Synthesis and Characterization of New Superconductors Materials
}

\author{
Edmondo Gilioli *(D) and Davide Delmonte *(D) \\ IMEM-CNR, Institute of Materials for Electronic and Magnetism-National Research Council, \\ Parco Area delle Scienze 37/A, 43124 Parma, Italy \\ * Correspondence: edmondo.gilioli@cnr.it (E.G.); davide.delmonte@imem.cnr.it (D.D.)
}

Received: 28 July 2020; Accepted: 28 July 2020; Published: 29 July 2020

In the last few decades, the persisting scientific interest in high temperature superconductor (HTS) cuprates has been accompanied by the search for new families of superconducting compounds (SPCs). Notable examples are the intermetallic borides (e.g., $\mathrm{MgB}_{2}$ ), iron-nickel-based superconductors (e.g., $\mathrm{La}(\mathrm{Fe}, \mathrm{Ni})(\mathrm{Pn}, \mathrm{Ch}) \mathrm{O},(\mathrm{Pn}, \mathrm{Ch}=$ pnictide or chalcogenide ions), heavy fermions (e.g., $\mathrm{CeCoIn} 5$ ), organic (doped-fullerene, e.g., $\mathrm{Rb}_{3} \mathrm{C}_{60}$, or fulvalene-based, e.g., (BEDT-TTF) ${ }_{2} \mathrm{X}$ ), superhydrides systems (e.g., $\mathrm{H}_{3} \mathrm{~S}$ ), etc., which strongly revitalized the research in this field.

Historically, the synthesis of new SPCs has played a crucial role; with the dream of increasing the critical temperature (Tc), a number of new SPC phases, as well as chemical substitutions or structural modifications induced by external stimuli (such as high pressures or magnetic field) of known compounds have been obtained. This massive effort still leads to important achievements: (i) on a more fundamental level, to refine and sometimes to develop theoretical models to explain the intriguing phenomenon of the superconductivity, (ii) to understand the correlation between structural and transport properties in the matter and (iii) to improve the SPC's properties in view of best performing practical applications.

The Special Issue "Synthesis and Characterization of New Superconductors Materials" of Crystals has been conceived to address various issues, providing reviews and insights on the most promising systems, as well as innovative solutions for practical applications.

K. Zhou et al. [1] systematically reviewed the crystal and electronic structure, the chemical substitution and the pressure- and thickness-dependent of FeSe-based SPC. Among the most interesting results reported: (i) the SPC properties vs. chemical substitution on the Fe or Se sites, (ii) the role of the application of high pressure in enhancing $T_{\mathrm{c}}$ and simultaneously induces magnetism, (iii) the organic and inorganic layer intercalated-FeSe leading to a $T_{\mathrm{c}}$ increase from 30 to $46 \mathrm{~K}$ and (iv) a further Tc increase due to the electron-phonon coupling between single FeSe unit cell and $\mathrm{SrTiO}_{3}$ substrate.

M. van Delft et al. [2] showed a clear evidence of a two- and three-dimensional superconducting phases in type-I Weyl semi-metals tantalum phosphide (TaP); remarkably, they revealed Tc between 1.7 and $5.3 \mathrm{~K}$ in as-grown TaP single crystals at ambient pressure, while all the known reports only found SPC through the surface contact with a sharp tip or the application of high pressure. They also showed how the Weyl semi-metals and other topological phases can host exotic phenomena such as non-zero momentum pairing or the Majorana fermion, a viable candidate for the ultimate realization of a scalable quantum computer.

T. Tani et al. [3] studied the field-angle dependence of interlayer magnetoresistance in crystals of organic conductor $\alpha$ (BEDT-TTF $)_{2} \mathrm{I}_{3}$ grown by the electrolysis method, under strong magnetic field. The authors demonstrated a qualitative agreement between the theory and experimental results of the field-angle dependence in this system. Besides, the quantum Hall ferromagnetic state assumed in the present study supports the helical surface state. 
HTS cuprates with perovskite structure are still the most widely studied SPCs; although the "original" compounds cannot be defined as new materials, a variety of unconventional chemical substitutions or treatments are reported to address either fundamental or practical issues.

Cabassi et al. [4] presented the role of chemical substitutions on (Bi,Pb)-2212 (BSCCO) HTS. They correlated the Tc and the structural morphology of $\mathrm{Zn}, \mathrm{Y}, \mathrm{Ti}$, and $\mathrm{Nd}$ chemically substituted compounds. Significantly, the elements are incorporated in the BSCCO-2212 structure at amounts exceeding the ranges currently found in the literature. In a few samples, the appearance of higher Tc is associated with the formation of BSCCO-2223.

N. Mohd Hapipi et al. [5] reported a comparative study on the AC susceptibility of $\mathrm{YBa}_{2} \mathrm{Cu}_{3} \mathrm{O}_{7-\delta}$ (YBCO) added with $\mathrm{BaZrO}_{3}(\mathrm{BZO})$ nanoparticles prepared via solid-state and co-precipitation method. Besides providing an analysis of the correlation between $\mathrm{BZO}$ concentration and $\mathrm{YBCO}$ grain size, the susceptibility measurement showed a decrease in the Tc onset with BZO addition, attributed to the distribution of $\mathrm{BZO}$ particles at the grain boundaries.

Z. Zhang et al. [6] optimized the synthesis of $\mathrm{ErBa}_{2} \mathrm{Cu}_{3} \mathrm{O}_{7-\delta}(\mathrm{Er}-123)$ to be used as a SPC joint between commercial a $\mathrm{GdBa}_{2} \mathrm{Cu}_{3} \mathrm{O}_{7-\delta}(\mathrm{Gd}-123)$-coated conductor. They systematically studied the effect of sintering parameters on the phase formation, microstructure and superconducting properties of Er-123 powder to obtain a high performing SPC joint. This work aims to improve the development of applications requiring long length HTS coated conductors.

A waiting for new exciting discoveries on superconductivity, we hope that the scientific community enjoys reading this Special Issue!

Funding: This research received no external funding.

\section{References}

1. Zhou, K.; Wang, J.; Song, Y.; Guo, L.; Guo, J. Highly-Tunable Crystal Structure and Physical Properties in FeSe-Based Superconductors. Crystals 2019, 9, 560. [CrossRef]

2. Van Delft, M.; Pezzini, S.; König, M.; Tinnemans, P.; Hussey, N.; Wiedmann, S. Two- and Three-Dimensional Superconducting Phases in the Weyl Semimetal TaP at Ambient Pressure. Crystals 2020, 10, 288. [CrossRef]

3. Tani, T.; Tajima, N.; Kobayashi, A. Field-Angle Dependence of Interlayer Magnetoresistance in Organic Dirac Electron System $\alpha$-(BEDT-TTF)2I3. Crystals 2019, 9, 212. [CrossRef]

4. Cabassi, R.; Delmonte, D.; Abbas, M.; Abdulridha, A.; Gilioli, E. The Role of Chemical Substitutions on Bi-2212 Superconductors. Crystals 2020, 10, 462. [CrossRef]

5. Mohd Hapipi, N.; Lim, J.; Chen, S.; Lee, O.; Shaari, A.; Awang Kechik, M.; Lim, K.; Tan, K.; Murakami, M.; Miryala, M. Comparative Study on AC Susceptibility of YBa2Cu3O7- $\delta$ Added with BaZrO3 Nanoparticles Prepared via Solid-State and Co-Precipitation Method. Crystals 2019, 9, 655. [CrossRef]

6. Zhang, Z.; Wang, L.; Liu, J.; Wang, Q. Synthesis of ErBa2Cu3O7- $\delta$ Superconductor Solder for the Fabrication of Superconducting Joint between Gdba2cu3o7- $\delta$ Coated Conductor. Crystals 2019, 9, 492. [CrossRef] 